\title{
Left Ventricular Segmentation in MR Using Hierarchical Multi-class Multi-feature Fuzzy Connectedness
}

\author{
Amol Pednekar ${ }^{1}$, Uday Kurkure ${ }^{1}$, Raja Muthupillai ${ }^{2}$, Scott Flamm , and \\ Ioannis A. Kakadiaris ${ }^{1 \star}$ \\ 1 Visual Computing Lab, Dept. of Computer Science, \\ University of Houston, Houston, TX, USA \\ 2 Philips Medical Systems North America, Bothell, WA, USA \\ 3 Dept. of Radiology, St. Luke's Episcopal Hospital, Houston, TX, USA
}

\begin{abstract}
In this paper, we present a new method for data-driven automatic extraction of endocardial and epicardial contours of the left ventricle in cine bFFE MR images. Our method employs a hierarchical, multi-class, multi-feature fuzzy connectedness framework for image segmentation. This framework combines image intensity and texture information with anatomical shape, while preserving the topological relationship within and between the interrelated anatomical structures. We have applied this method on cine bFFE MR data from eight asymptomatic and twelve symptomatic volunteers with very encouraging qualitative and quantitative results.
\end{abstract}

\section{Introduction}

Magnetic resonance imaging (MRI) is the preferred cardiac imaging technique as it acquires images in oblique planes; obviating geometric assumptions regarding the shapes of the ventricles providing high blood-to-myocardium contrast. However, the cine cardiac MR (CMR) images are not incisive; they are fuzzy due to patient motion, background variation, and partial voluming. Left ventricle (LV) myocardial delineation allows the computation of critical LV functional descriptors (e.g., ejection fraction and wall thickening). The manual contour tracing used in current clinical practice is labor-intensive, time-consuming, and involves considerable inter- and intra-observer variations [1]. The development of computer-assisted myocardial contour extraction will reduce the analysis time and, more importantly, will produce unbiased and consistent results. The segmentation of CMR data typically faces two challenges: the determination of the inter-tissue boundaries (blurred due to partial voluming), and the delineation of

\footnotetext{
* This material is based upon work supported in part by the National Science Foundation under Grants IIS-9985482 and IIS-0335578. Any opinions, findings, and conclusions or recommendations expressed in this material are those of the authors and do not necessarily reflect the views of the National Science Foundation.
} 
the boundaries of anatomical structures composed of the same tissue type (e.g., trabeculae carnae and papillary muscles projecting out of the myocardium).

Most of the research towards automated segmentation of CMR data follows one of three major approaches: 1) active contour-based methods - positioning of a two-dimensional (2D) contour near the strongest local image features using the principle of energy minimization [23] (these methods rely on user interaction for initialization of the shape and location of the ventricle's boundaries); 2) deformable model-based methods - three-dimensional (3D) shape fitting for functional analysis of cardiac images using physics-based deformable models [4,5] 6] ; and 3) active appearance-based methods - fitting with models that provide a statistical description of local shape (geometry) and appearance (brightness) [7] 8] (these methods could be biased towards a "too normal" pattern of the LV shape and its dynamics). Research is ongoing in developing hybrid segmentation methods for the extraction of LV endocardial boundary surfaces by combining edge, region, and shape information [9|10 1112]. Our hybrid segmentation approach is data-driven and hence works across a wide range of patient data. It is an extension of the fuzzy connectedness-based image segmentation framework developed by Udupa and Samarasekera [13], which effectively captures the fuzzy "hanging togetherness" of image elements specified by their strength of connectedness. That framework has been further extended with the introduction of object scale, relative strength of connectedness, multiple objects, and multidimensional images. The idea of using dynamic adaptive weights for affinity components was introduced in our previous work [14].

In this paper, we present a new method for segmenting the LV myocardium automatically. The main steps are the following: 1) automatic localization of the LV and myocardium using multi-view, intensity, and shape information; 2) employing a hierarchical, multi-class, multi-feature fuzzy connectedness framework to overcome the low contrast between interfacing tissues; 3) integrating shapeoriented coordinate transforms, myocardial fuzzy affinity, and optimal path computation using dynamic programming to overcome anatomy-specific challenges (such as those posed by papillary muscles projecting out of the myocardium). Our data-driven method does not require any user interaction at either the LV identification or the myocardial segmentation phases. Our contributions include the development of a hierarchical, multi-class, multi-feature fuzzy connectedness framework for image segmentation, which combines the image intensity and texture information with the shape of the anatomy, while preserving the topological relationship within and between the interrelated anatomic structures. The main attributes of this framework are the following: 1) computing multi-class affinity that takes into consideration the affinity component distributions of all the neighboring objects (which allows competition between different objects); 2) computing multi-feature affinity; and 3) integrating multi-class affinity and multi-feature affinity components to compute fuzzy affinity, where the weights for individual affinity components are determined automatically. 


\section{Method}

In this section, we present our hierarchical, multi-class, multi-feature fuzzy connectedness framework and its application to the segmentation of the LV myocardium.

\subsection{Hierarchical Multi-class Multi-feature Fuzzy Connectedness}

We describe our formulation of hierarchical, multi-class multi-feature fuzzy connectedness using the terminology introduced by Udupa and Samarasekera [13. The aim of image segmentation is to capture the local and global "hangingtogetherness" that is, the perception of a coherent object region in spite of heterogeneity of scene intensity) of pixels. For any relationship $\rho$, the strength of $\rho$ between any two pixels $c$ and $d$ in digital space $Z^{2}$ is represented by a membership function $\mu_{\rho}(c, d)$.

Local Fuzzy Spel Affinity: Any fuzzy relation $\kappa$ in a scene domain $C$ is a fuzzy pixel affinity in a scene $\mathcal{C}$ if it is reflexive and symmetric. We define the local fuzzy spel affinity $\left(\mu_{\kappa}\right)$ to consist of three components: 1) the object feature intensity component $\left.\left(\mu_{\phi}\right) ; 2\right)$ the intensity homogeneity component $\left(\mu_{\psi}\right)$; and 3 ) the texture feature component $\left(\mu_{\varphi}\right)$. This can be expressed in the following form:

$$
\mu_{\kappa}(c, d)=\mu_{\alpha}(c, d) g\left(\mu_{\phi}(c, d), \mu_{\psi}(c, d), \mu_{\varphi}(c, d)\right)
$$

where $\mu_{\alpha}(c, d)$ is a hard adjacency relation. Thus, the fuzzy relation $\kappa$ in a digital space $Z^{2}$ indicates the degree of local hanging togetherness of pixels $c$ and $d$ in the space of the feature vector:

$$
\mathbf{x}=\left[\frac{1}{2}(f(c)+f(d)), f(c)-f(d), \frac{1}{2}(t(c)+t(d))\right]^{\top}
$$

where $f(c)$ and $f(d)$ are the image intensities, and $t(c)$ and $t(d)$ are the texture features at pixels $c$ and $d$. The similarity of the pixels' feature vectors is computed using the Mahalanobis metric:

$$
m_{d(\mathbf{c} \rightarrow \mathbf{d})}^{2}=\left(\mathbf{x}_{(\mathbf{c} \rightarrow \mathbf{d})}-\overline{\mathbf{x}}_{(\mathbf{c} \rightarrow \mathbf{d})}\right)^{\top} S_{(\mathbf{c} \rightarrow \mathbf{d})}^{-1}\left(\mathbf{x}_{(\mathbf{c} \rightarrow \mathbf{d})}-\overline{\mathbf{x}}_{(\mathbf{c} \rightarrow \mathbf{d})}\right),
$$

where $\mathbf{x}_{(\mathbf{c} \rightarrow \mathbf{d})}, \overline{\mathbf{x}}_{(\mathbf{c} \rightarrow \mathbf{d})}, S_{(\mathbf{c} \rightarrow \mathbf{d})}$ are the feature vector, the mean feature vector, and the covariance matrix in the direction from $c$ to $d$, respectively. The bias in intensity in a specific direction is accounted for by allowing different levels and signs of intensity homogeneities in different directions of adjacency [14]. The advantage of using the Mahalanobis metric is that it weighs the differences in various feature dimensions by the range of variability in the direction of the feature dimension. These distances are computed in units of standard deviation from the mean. This allows us to assign a statistical probability to the measurement. The local fuzzy spel affinity is computed as: $\mu_{\kappa}(c, d)=\frac{1}{1+m_{d(\mathbf{c} \rightarrow \mathbf{d})}}$ to ensure $\mu_{\kappa}(c, d) \in Z^{2} \rightarrow[0,1]$ and it is reflexive and symmetric. 
Global Object Affinity: Fuzzy connectedness captures the global hangingtogetherness of pixels by using the local affinity relation and by considering all possible paths between two, not necessarily nearby, pixels in the image. Fuzzy $\kappa$ connectedness in $\mathcal{C}$, denoted $K$, is a fuzzy relationship in $C$ that assigns to every pair $(c, d) \in C$ a value $\mu_{K}(c, d)=\max _{p_{c d} \in P_{c d}}\left\{\min _{1 \leq i \leq m}\left[\mu_{\kappa}\left(c_{(i)}, c_{(i+1)}\right)\right]\right\}$, which is the largest of the weakest affinities between the successive pairs of pixels along the path $p_{c d}$ of all possible paths $P_{c d}$ in $\mathcal{C}$ from $c$ to $d$. For any seed pixel $o \in C$, the $\kappa$-connectivity scene of $o$ in $\mathcal{C}$ is the scene $\mathcal{C}_{K_{o}}=\left(C, f_{K_{o}}\right)$ such that, for any $c \in C, f_{K_{o}}(c)=\mu_{K}(o, c)$. For any strength $\theta \in[0,1]$, the binary relationship defined by $\mu_{K}(c, d) \geq \theta$, denoted $K_{\theta}$, and defined in $C$ is an equivalence relation in $C$ [13. For any seed pixel $o \in C$, let $O_{K \theta}(o)$ be an equivalence class of $K_{\theta}$ in $C$ that contains $o$. A fuzzy $\kappa \theta$-object of $o$ in $\mathcal{C}$ is a fuzzy subset $O_{K \theta}(o)$ of $C$ such that, for any $c \in C$

$$
\mu_{O_{K \theta}(o)}(c)=\left\{\begin{array}{ll}
\eta(f(c)) & \text { if } \mu_{K}(o, c) \geq \theta \\
0 & \text { otherwise }
\end{array},\right.
$$

where $\eta$ is an objectness function with range $[0,1]$. Since $\mu_{\kappa}(c, d)=\frac{1}{1+m_{d(\mathbf{c} \rightarrow \mathbf{d})}}$, $\theta \leq 0.25$ denotes a probability of 0.01 or less and can be used to define the binary object. Thus, $\mu_{\kappa}(c, d)$ defines the probability of pixel pair belonging to the target object class. The threshold for the object definition can be set based on the probability distribution in a specific feature space for a particular application.

Global Class Affinity: In our framework the global object affinity and local pixel affinity are assigned only if the probability of $c$ and $d$ belonging to the neighboring objects' classes is much less than 0.01 . The neighboring objects are defined as the objects with common boundaries in Euclidean space. For a given pixel pair $(c, d)$, we compute the discrepancy measure with respect to the predetermined or existing distributions (covariance matrices) of neighboring classes in terms of its Mahalanobis distance. Then, the minimum discrepancy measure $J(c, d)=\min _{1 \leq i \leq b} m_{d}(c, d)$, where $b$ is the number of neighboring classes of the target object, gives the maximum probability of a pixel pair belonging to a certain class. If the $J(c, d)<3$, and the class to which the pixel pair belongs is not the target object class, then local pixel affinity $\mu_{\kappa(c, d)}=0$, else the pixel pair is a candidate to be considered to belong to the target object, hence its local pixel and global object affinity is computed as described earlier.

\subsection{Left Ventricular Segmentation in MR}

To apply our hierarchical, multi-class, multi-feature fuzzy connectedness algorithm for LV segmentation, first we determine the relevant classes and features. The training is performed only once for this application. Next, we compute the target object seed pixel and sample regions, and extract the LV myocardial contours. We further refine the myocardial contours to overcome the papillary muscles by using dynamic programming on the polar representation of the data. 


\section{A. Determination of Relevant Classes and Features}

Step 1 - Compute the intensity and texture features: In the case of bFFE CMR data, the contrast between blood and myocardium is high. However, the tissue contrast between myocardium and air, and myocardium and liver is not adequate for intensity-based discrimination. Thus, we compute the Laws 15 and Gabor [16] texture features of these neighboring tissue types to increase the discrimination. Laws texture features are generated by using the $2 \mathrm{D}$ convolution kernels obtained by convolving five one-dimensional (1D) kernels with one another. Gabor features are obtained by filtering the original images with filter banks of four scales and six orientations of quazi-orthogonal Gabor filters. Currently, we employ the data from 10 subjects using three mid-ventricular enddiastolic (ED) slices per subject. In these 30 images, we manually delineated the myocardium, blood, liver, and air.

Step 2 - Compute the most discriminant texture features: From the feature images, we extract pixel features for the following classes: myocardium, blood, liver, and air. The texture features are ranked according to their individual ability to discriminate myocardium from all the neighboring tissues using the Fisher's criterion and the Mahalanobis distance measure. We found that spotspot (ss) and spot-average intensity level (sl) Laws features are individually the most discriminating. Laws spot and level 1D kernels represent a second derivative filter and an averaging filter, respectively. The convolution of spot with spot, and spot with level kernels provide the ss and sl filters. Further, we found that combining intensity and intensity gradient with the ss feature provided higher discrimination between myocardium and other neighboring tissues. However, the discrimination between the myocardial muscle and the liver tissue remains low because the $T 2$ values of these tissues are very similar. Thus, our feature vector (Eq. (21) ) consists of pixel pair intensity, directional gradients, and the ss feature in a $5 \times 5$ neighborhood.

\section{B. Extraction of the LV Myocardial Contours}

Step 1 - Determine the LV medial axis: First, we determine the LV centroids in the blood region. In CMR imaging, the short-axis view is planned from vertical long-axis, two chamber $(2 \mathrm{CH})$, and approximate four chamber $(4 \mathrm{CH})$ scouts.

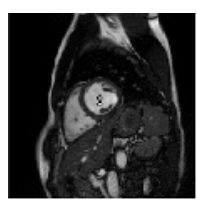

(a)

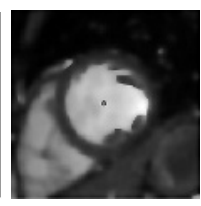

(b)

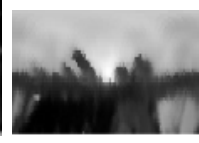

(c)

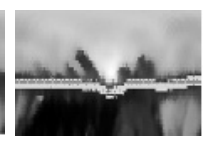

(d)

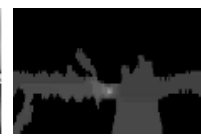

(e)

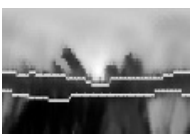

(f)

Fig. 1. (a) Original MRI (with LV center localization), (b) cropped (with LV center localization), and (c) polar image of mid-ventricular slice of Subject-1. Corresponding (d) sample region, (e) affinity image, and (f) endo- and epicardial contours for myocardium. 
This multi-view information along with intensity, shape, and temporal information is used to localize the 3D medial axis of the LV. Specifically, we map the end-diastolic (ED) lines corresponding to the $2 \mathrm{CH}$ and the $4 \mathrm{CH}$ scouts onto the ED short-axis as a cross-hair. Due to patient motion between acquisitions and the curved nature of the LV, the cross-hair does not necessarily fall into LV in all the slices. We then crop the ED short-axis images around the cross-hair intersection point (Fig. 1(b)). Thresholding the cropped images using the threshold provided by Otsu's algorithm 17 provides the blood regions. This threshold is obtained by maximizing a function of the ratio of the between-class variance and the total variance of the intensity levels. The LV region is extracted from the binary image using the cost function based on the larger blood area and the minimum distance of the center of blood regions from the cross-hair. The LV centroids detected in each slice are then updated using a cost function which maintains 3D continuity of the LV medial axis along the entire volume from base to apex. These ED LV medial axis points are then propagated along time as the initial LV centroids in all the phases, and they are then updated using the above cost function to maintain the temporal continuity of the LV medial axis. Figures 2 $(a, b)$ depict the estimated centroids in the ED and the ES phases for Subject-1.

Step 2 - Compute myocardium seed pixel and sample regions: In the second step, we determine the seed point for the LV myocardium and the sample regions for all the classes. In cine CMR data, tissue types (due to their feature responses) and LV myocardium (due to its spatial adjacency in polar coordinates) form clusters in a feature space, thus providing clues for LV blood and myocardium classification. We convert images into polar coordinates (Fig. 11(c)) and form the feature space of $(p, i)$ (Fig. 2(c)). The $p$ coordinate is formed by appending rows of pixels in the polar image according to their radius value. We use subtractive clustering [18] in the $(p, i)$ space to identify the different clusters. Starting from zero radius, the first centroid with the highest intensity value and the last centroid with the lowest intensity value are identified as the centroids for the LV blood and the air clusters, respectively. Next, we take the derivative of the line connecting the centroids of the detected clusters with respect to the intensity and the first negative peak from zero radius is identified as the centroid for the myocardial cluster. The intensity values in these clusters along with their position provide the myocardial seed pixel with the sample region (Fig. 1(d)) and the feature distribution for all the classes.

Step 3 - Segmentation: Having determined the feature vector distributions and seed regions for LV blood, myocardium and air, we segment the LV myocardium using our framework. The segmented region includes the papillary muscles and occasionally the liver. Thus, we employ dynamic programmingbased border detection in polar coordinates on the segmented region to exclude the papillary muscles and obtain the endocardial and epicardial contours (Figs. 1] e,f)) [12]. 

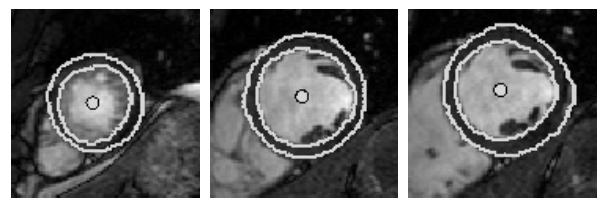

(a)
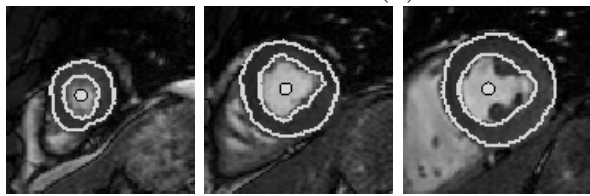

(b)

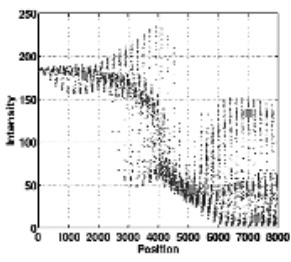

(c)

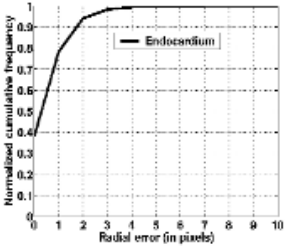

(d)

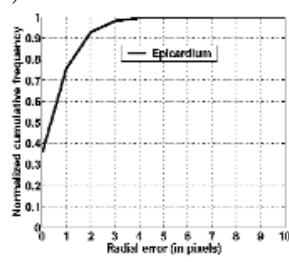

(e)
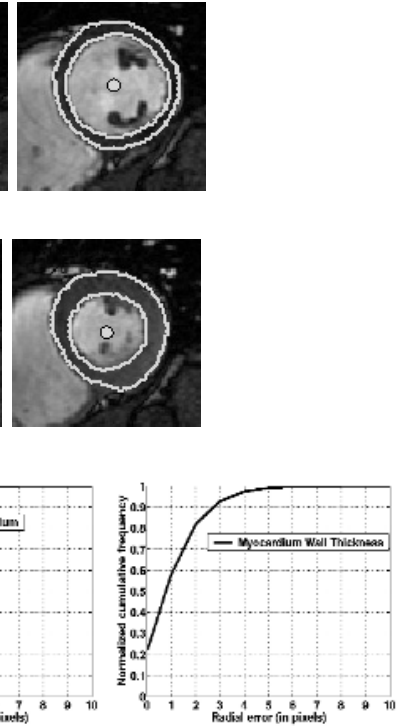

(f)

Fig. 2. The automatically estimated centroids and myocardial contours in (a) ED and (b) ES slices for Subject-1. (c) Cluster centers in $(p, i)$. Cumulative distribution of radial distance error for (d) endocardium, (e) epicardium, and (f) wall thickness.

\section{$3 \quad$ Results}

We applied our framework for extracting the LV myocardial contours in three mid-ventricular ED slices of 20 subjects. Volunteers were imaged on a $1.5 \mathrm{~T}$ commercial scanner (Philips Gyroscan NT-Intera) using vector-cardiographic gating. The bFFE short-axis sequence was acquired to cover the entire LV. The acquisition parameters for a cine bFFE sequence were TE/TR/flip: 3.2/1.6/55 deg; 38-40 msec temporal resolution. The endocardial and epicardial contours determined by our algorithm for Subject-1 are depicted in Figs. 2(a,b). We validated our results against the endocardial and epicardial contours drawn manually by experts from St. Luke's Episcopal Hospital. The automatically detected contours are quantitatively assessed for the border positioning error in terms of radial distance of each point from the corresponding point on the manually traced contour. The radial error magnitude effectively captures the effect of the error on the LV volume, EF, and WT computations. Figures 2/(d-f) depict the cumulative radial distance errors between automatic and manual contours. Note that maximum error of three or more pixels for endo- and epicardial contours and five pixels for wall thickness are observed less than $1 \%$ of the time.

\section{Conclusion}

Our data-driven method does not require any user interaction at either the LV identification or the myocardial segmentation phases. This method allows accu- 
rate extraction of endocardial and epicardial contours of the $\mathrm{LV}$ in cine bFFE MR images. The results of our algorithm are consistent with the manual tracings of clinical experts. This method allows unbiased and consistent computation of the following LV functional parameters: cardiac output, ejection fraction, end-diastolic and end-systolic volumes, stroke volume, wall thickness, and wall thickening.

\section{References}

1. Matheijssen, N., Baur, L., Reiber, J., der Velde, E.V., Dijkman, P.V., der Geest, R.V., de Ross, A., der Wall, E.V.: Assessment of left ventricular volume and mass by cine magnetic resonance imaging in patients with anterior myocardial infarction: Intra-observer and inter-observer variability on contour detection. International Journal of Cardiac Imaging 12 (1996) 11-19

2. Paragios, N.: Shape-based segmentation and tracking in cardiac image analysis. IEEE Transactions on Medical Imaging 22 (2003) 773-776

3. Lelieveldt, B., van der Geest, R., Rezaee, M.R., Bosch, J., Reiber, J.: Anatomical model matching with fuzzy implicit surfaces for segmentation of thoracic volume scans. IEEE Trans. on Medical Imaging 18 (1999) 218-230

4. Frangi, A., Niessen, W., Viergever, M.: Three-dimensional modeling for functional analysis of cardiac images: A review. IEEE Trans. Med. Imaging 20 (2001) 2-25

5. Singh, A., Goldgof, D., Terzopoulos, D.: Deformable Models in Medical Image Analysis. IEEE Computer Society, Los Alamitos, CA (1998)

6. Park, J., Metaxas, D., Young, A., Axel, L.: Deformable models with parameter functions for cardiac motion analysis from tagged MRI data. IEEE Trans. Medical Imaging 15 (1996) 278-289

7. Mitchell, S., Bosch, J., Lelieveldt, B., van der Geest, R., Reiber, J., Sonka, M.: 3-D active appearance models: segmentation of cardiac MR and ultrasound images. IEEE Trans. on Medical Imaging 21 (2002) $1167-1178$

8. Sonka, M., Lelieveldt, B., Mitchell, S., Bosch, J., der Geest, R.V., Reiber, J.: Active appearance motion model segmentation. In: Second International Workshop on Digital and Computational Video, Tampa, Florida, (2001) 64-68

9. Paragios, N.: A variational approach for the segmentation of the left ventricle in cardiac image analysis. Int. Journal of Computer Vision 50 (2002) 345-362

10. Jolly, M.: Combining edge, region, and shape information to segment the left ventricle in cardiac MR images. In: Proc. of the 4th Int. Conference on Medical Image Computing \& Computer-Assisted Intervention, Utrecht, The Netherlands, (2001) 482-490

11. Imielinska, C., Metaxas, D., Udupa, J., Jin, Y., Chen, T.: Hybrid segmentation of anatomical data. In: Proc. of the 4th Int. Conference on Medical Image Computing \& Computer-Assisted Intervention, Utrecht, The Netherlands, (2001) 1048-1057

12. Pednekar, A., Kakadiaris, I., Kurkure, U., Muthupillai, R., Flamm, S.: Intensity and morphology-based energy minimization for the automatic segmentation of the myocardium. In: Proc. of IEEE Workshop on Variational and Level Set Methods, Nice, France, (2003) 185-192

13. Udupa, J., Samarasekera, S.: Fuzzy connectedness and object definition: theory, algorithms, and applications in image segmentation. Graphical Models and Image Processing 58 (1996) 246-261 
14. Pednekar, A.S., Kakadiaris, I., Kurkure, U.: Adaptive fuzzy connectedness-based medical image segmentation. In: Proceedings of the Indian Conference on Computer Vision, Graphics, and Image Processing, Ahmedabad, India (2002) 457-462

15. Laws, K.: Textured Image Segmentation. PhD thesis, USC (1980)

16. Manjunath, B.S., Ma, W.Y.: Texture features for browsing and retrieval of image data. IEEE Trans. Pattern Anal. Mach. Intell. 18 (1996) 837-842

17. Otsu, N.: A threshold selection method from gray-level histograms. IEEE Transactions on Systems, Man, and Cybernetics SMC-9 (1979) 62-66

18. Chiu, S.: Fuzzy model identification based on cluster estimation. Journal of Intelligent Fuzzy Systems 2 (1994) 267-278 